\title{
Why under five children are stunted in Pakistan? A multilevel analysis of Punjab Multiple indicator Cluster Survey (MICS- 2014)
}

Tahir Mahmood ${ }^{1,2}$, Faisal Abbas $^{3}$ (D) Ramesh Kumar $^{4,5^{*}}$ (D) and Ratana Somrongthong ${ }^{5^{*}}$ (D)

\begin{abstract}
Background: Pakistan is facing a serious problem of child under-nutrition as about 38\% of children in Pakistan are stunted. Punjab, the largest province by population and contributes high gross domestic product (GDP) share in economy has reported $27 \%$ moderately and $10 \%$ severely stunted children of less than 5 years. Thus, this study aims at examining the determinants of stunting (moderate and severe) at different level of hierarchy empirically in Punjab province of Pakistan.
\end{abstract}

Methodology: Data for this study is coming from Punjab Multiple Indicators Cluster Survey (MICS-2014), used twostage, stratified cluster sampling approach. Sub-national level data covering urban and rural areas were used for this study consists of 25,067 children less than 5 year's ages, from nine administrative divisions and 36 districts of Punjab province of Pakistan. Descriptive statistics and multilevel hierarchical models were estimated. Multilevel data analyses have an advantage because it provides robust standard error estimates and helps in finding variation in the data at various levels.

Results: Punjab has a stunting prevalence of about $27 \%$ moderately and $10 \%$ severely stunted children of less than 5 years. The results depict that increasing the age of the child, increasing birth order, illiterate mothers and fathers, lack of sanitation facilities and being poor are associated significantly with the likelihood of moderate and severe stunting. Surprisingly, there is a gender bias in stunting in Punjab, Pakistan and being a girl child is more likely associated with moderate and severe stunting, which shows the patriarchal nature of the society and a substantial prevalence of gender bias in household resource allocations.

Conclusion: This outcome of our analysis points towards targeting not only households (focus on girls) but also their families and communities.

Keywords: Undernutrition, Stunting, Child health, Pakistan, Punjab, Multilevel models, Multiple indicators cluster survey

\footnotetext{
* Correspondence: drramesh1978@gmail.com; sratana3@chula.ac.th

${ }^{4}$ Health Systems \& Policy Department, Health Services Academy, Islamabad,

Pakistan

${ }^{5}$ College of Public Health Sciences, Chulalongkorn University, Bangkok,

Thailand

Full list of author information is available at the end of the article
}

(c) The Author(s). 2020 Open Access This article is licensed under a Creative Commons Attribution 4.0 International License, which permits use, sharing, adaptation, distribution and reproduction in any medium or format, as long as you give appropriate credit to the original author(s) and the source, provide a link to the Creative Commons licence, and indicate if changes were made. The images or other third party material in this article are included in the article's Creative Commons licence, unless indicated otherwise in a credit line to the material. If material is not included in the article's Creative Commons licence and your intended use is not permitted by statutory regulation or exceeds the permitted use, you will need to obtain permission directly from the copyright holder. To view a copy of this licence, visit http://creativecommons.org/licenses/by/4.0/. The Creative Commons Public Domain Dedication waiver (http://creativecommons.org/publicdomain/zero/1.0/) applies to the data made available in this article, unless otherwise stated in a credit line to the data. 


\section{Background}

One out of five children under 5 year's age is reported stunted and contributes half of the child mortality in the world [1].. Globally, more than half (56\%), 87 million of the total stunted children are living in South Asia alone. A stunted child is physically short of their age and thus fails to grow cognitively. This condition of undernourishment of a child undermines their potential of life from its very manifestation and affects over the life cycle [2]. Under-nutrition leads to child morbidity and mortality, poor physical and cognitive development, poor school performance, reduced capacity to work even later in life, thus loss of productivity and wages [3-5]. Therefore, under-nutrition is one of the most pressing challenges of the contemporary era due to its long-lasting and detrimental consequences [6].

Moreover, inadequate nutrition of the mother during pregnancy and in the first 2 years of a child's life is an important window of opportunity to realize a child's full potential of life. If this window of opportunity is missed could cause irreversible and retarded growth, impaired learning and reduced work performance [2]. Stunting refers to poor child health status due to multiple factors like; physical development, cognitive abilities and the environment [7-11]. Stunting is the form of undernutrition (reduced height for age) evaluated by using anthropometric indices as a proxy measure according to WHO child growth standards [12, 13]. After India, Pakistan has the highest number of stunted children in South Asia [14-16]. Moreover, malnutrition is widespread in Pakistan among all age groups and the progress is not promising in the last decades $[17,18]$.

In Punjab, stunting prevalence in the year 2011 was $36 \%$ and in 2014 , it is reduced to $33.5 \%$, this prevalence rate of stunting is alarming for the largest and most developed province of the country. Punjab contributes more than $52 \%$ to the national income and about $56 \%$ of the population of the country resides in this province. Given the severe prevalence of stunting in one of the largest province of Pakistan, this study investigates in detail the prevalence and determinants of stunting in Punjab as an important research question. Moreover, it is imperative to analyze the factors, i.e., immediate (Child level characteristics), underlying (mother, father, household and community level characteristics) and basic (i.e., areas of residence, the region of residence and regional food security status) associated with stunting in Punjab, Pakistan [13].

The main factors associated with stunting according to literature are; multiple births, low birth-weight, maternal education, household wealth, maternal body mass index and the short birth interval [19]. Similarly, previous studies shows that an individual and community-level factors are important determinants of childhood stunting and confirm that $37 \%$ of stunting is due to community- level factors in their study [20, 21]. Apart from child specific characteristics and parental characteristics, the importance of water, sanitation and hygiene (WASH) have been recognized regarding health of infants and young children, particularly when the process of stunting is concentrated during the first 2 years of life [10].

Recent studies show that the poor diet of children in the first year of life, poor nutritional status of a mother during and after pregnancy, poor sanitation practices, food insecurity and poverty, and social inequalities are significant drivers of childhood stunting [22-24]. In addition, child nutritional status is commonly influenced by factors such as adequate health services and/or lack thereof, nutritional and social services facilities at community/village level and access to these facilities [25-28]. Another study found that safe water access, education of women, gender equality, and food availability are the main drivers in reducing stunting [1].

Although, literature on Pakistan is rather scant [18]. There are attempts to investigate the determinants of under-nutrition not only at the national level but also at the micro-geographic level [29-34]. A study from Sindh province also pinpoints mother illiteracy, low income, and overcrowding as significant risk factors of stunting [30]. Additionally, education among rural mothers could reduce stunting, as being female are more likely to be stunted and underweight compared to male in these areas [31]. Contrary to this assertion, for Punjab, a study using MICS 2011 data explored that female headedhousehold (a measure of female empowerment) had 26\% lower odds of childhood stunting [34].

All these research studies have focused on employing either ordinary least square (OLS) or binary logistic regression analyses. As standard OLS may over or underestimate the coefficient of correlates at different points across the distribution of stunting prevalence, thus, bias the estimates. These hierarchies have vital information to explore for better and targeted policy formulation. Therefore, this study uses a multilevel hierarchical modeling approach to unravel the variation at household, community and the district level that is not explored in the case of Pakistan at the micro-geographic level. This analysis will set up the stage for policy level debate and provide the data-driven information for public policy design. This study is first to analyze the determinants of stunting in Punjab Pakistan using MICS-2014, which includes more than 31,000 children of less than 5 years [13].

\section{Methods}

The Multiple Indicator Cluster Survey (MICS) is an international household survey program developed by United Nations Children's Fund (UNICEF) to collect comparable estimates of more than $125 \mathrm{key}$ indicators that are used to assess the situation of children and women, in 
Punjab Pakistan. The MICS consists of all the households and their members in all urban and rural areas of Punjab, which is divided into 9 administrative divisions and 36 districts. A two-stage, stratified cluster sampling approach was used for the selection of the survey sample. The firststage selection unit is an enumeration and the village in urban and rural areas. From each of the first-stage sample units, a sample of 20 households selected both in rural and urban areas. Households were considered as secondary sampling units (SSUs) for urban and rural domains. The entire sample of households (SSUs) was drawn from 2050 Primary Sampling Units (PSUs), out of which 774 were urban and 1276 were rural, adopting a systematic sampling technique with a random start. The final recommended allocation is 2050 clusters with 20 households in each, giving a total sample of 41,000 households and the response rate is almost $98 \%$ across Punjab province. The detail on MICS Punjab 2014 is provided elsewhere [13].

The current study investigates the determinants of stunting of under five years of children. For this purpose, after cleaning the data, we end up with 25,066 children of under 5 year of age.

\section{Dependent variables}

Stunting (i.e., moderate and severe) is used as a binary variable with not stunted being coded as zero while being stunted coded as one, similar is the case with severe stunted. Stunted is measured as moderate if the height for age $\mathrm{z}$ score of a child less than 5 years is less than minus 2 standard deviation from WHO reference growth standards and it is severe if $\mathrm{z}$ score is less than minus 3 standard deviation [35].

\section{Independent variables}

Individual and household level characteristics include; the age of a child in months and is categorized into (0-5 months, 6-11 months, $12-23$ months, $24-35$ months, 3647 months and 48-59 months). We divided the first year of the child into two categories because for the first 6 months, a child is exclusively breast-fed or having milk only as his (her) diet. Moreover, between 6 and 12 months, he/she is starting solid food as well. Birth order of the child, this variable is a rank number of children born to women age 15-49 years and categorized into; first born, second born and third or after born having age less than 5 years. The gender of a child is either boy or girl. The size of the child at birth is divided into three categories, i.e. large (combining very large and larger than average birth size category), average and small (combining smaller than average and very small category). ${ }^{1}$ Diarrhea, whether

${ }^{1}$ If baby weighs $4500 \mathrm{~g}$ or more at birth, they are considered larger than normal. Low birth weight less than $2500 \mathrm{~g}$ (upto and including 2499g). Very low birth weight less than $1500 \mathrm{~g}$ (upto and including $1499 \mathrm{~g}$ ) a child had diarrhea in the last 2 weeks ( 15 days), is a binary variable (Yes $=1$; zero otherwise). Breastfeeding is a binary variable if a child is ever breastfed (Yes $=1)$ and $(\mathrm{No}=0)$.

Parental education is categorized as; no education (less than 1 year), primary ( $1-5$ years completed), middle (68 years of a complete education), secondary (9-10 years of a complete education) and higher $(11+$ years of complete education including professional or university degrees). Mother's age is a categorical variable; less than 18 years, $18-24$ years, $25-35$ years and $35+$ years. Postnatal care is proxies by whether a Lady Health Worker (LHW) visited the household in the past 3 months and is a binary variable. This variable captures the knowledge and information exchange that is positively associated with child health outcomes. Antenatal care visits (ANC visits) are the number of times a woman received antenatal care during pregnancy and is coded as; less than 4 times as recommended by WHO and 4 times or higher times. Place of delivery where a woman gives birth to her child is coded as home delivery if the birth occurred at her own home or another home and institutional delivery if the delivery occurred at either public or private hospital or maternity care or basic/primary health care center. Household size includes the number of household members in a household and is categorized into; $3-$ 4 household members, 5-6 household members which is an average size of a family in Punjab, 7-8 household members and more than 8 household members to capture the effect of a very large family on child health outcomes.

Ethnicity is asking about the mother tongue of the head of the household and is categorized into; Urdu, Punjabi, Saraiki and Others. Place of residence is a binary variable and coded as urban $=1$ and rural $=0$. Similarly, the sanitation facility is about the toilet facility a household possesses. It has been divided into two categories, according to the WHO definition. The first category is improved sanitation facility, which includes sewer connections, septic system connection, pour-flush latrines, ventilated improved pit latrines and pit latrines with a slab or covered pit. The second category is unimproved sanitation facility, which includes pit latrines without slabs or platforms or open-pit hanging latrines bucket latrines, open defecation in fields, forests and/or bushes. Water treatment (boiling water before drinking) is asking a household what household do to make water safer for drinking and is a binary variable coded as treated $=1$ and untreated $=0$.

Regional characteristics include districts wise food insecurity index and administrative divisions of the province of Punjab. The food insecurity index is taken from the SDPI report and this index is at the district level. All three composite indicators (availability, access and food 
absorption) were aggregated to determine the food insecurity level of the districts in Pakistan. The food insecurity indicator was not the simple sum of all composite indicators, but rather a principal component analysis (PCA) was used [22, 24, 36]. The food insecurity index is divided into three categories. (1) Food Insecure (0.530.71), (2) Vulnerable $(0.72-0.80,3)$ Food Secure (0.810.93). The measure of food insecurity helps in better understanding the variation at sub national level (district); hence this variable is included at the district level. Furthermore, the Punjab province is divided into nine administrative divisions; Rawalpindi, Bahawalpur, D. G. Khan, Faisalabad, Gujranwala, Lahore, Multan, Sahiwal and Sargodha.

\section{Data analysis}

The simultaneous analysis of two variables is captured through bivariate analysis. It explores whether there exists a significant difference between the variables or not. This study presents the prevalence of moderate and severe childhood stunting in percentages at various levels of covariates, the level of significance of each variable with that of the response variable set at P-value $<0.05$. In addition, the current study employs three-level random intercept multilevel hierarchical models. Many kinds of data, including observational data, have a hierarchical or clustered structure. For example, children with the same parents tend to be more alike in their physical attributes, social attitudes, and mental characteristics than children are chosen randomly. Multilevel models recognize the existence of data hierarchies by varying the residual components at each level in the hierarchy [37]. In a multilevel model, the groups in the sample are treated as a random sample, where it is not possible in the fixed-effect model. For instance, if we use ordinary regression analysis in finding factors associated with stunting, we assume that for all children, the outcomes (in this case, moderate and severe stunting) are independent. Nevertheless, in our analysis, more than one child per family is included so that outcomes for siblings may be correlated, especially if the nutritional status is dependent on characteristics common to a family such as the quality of parental care, the sanitary conditions within the household etc. Because of these household effects, we would expect stunting for children in the same household or community to be more alike than stunting for children from different households or communities [36]. A simple three-level model without the explanatory variables, which is called unconditional model is written as: Stunting $i j k=b_{0}+v_{k}+u_{j k}+e_{i j k}$ Where Stunting ijk $_{\text {is }}$ is the observed value of being (Stun$\left.t_{i n g_{i j k}}=1\right)$ both moderately and severely stunted and being non-stunted (Stunting ${ }_{i j k}=0$ ) child under-five in household $i$, in community $j$ and district $k$.
Several approaches are available to interpret variance components in multilevel models; we are considering here the; variance partition coefficients (VPCs) and intraclass correlation coefficients (ICCs). The VPC statistics report the proportion of the response variance that lies at each level of the model hierarchy. Thus, the total variance is simply the sum of the three separate variance components. The ICC measures the expected degree of similarity between responses within a specific level.

As the OR sometimes does not give a good approximation and does not provide information about the strength of the association (effect size), therefore, this study also calculates an important measure of effect size known as Cohen's $d$. Cohen suggested a threshold that $d=0.2$ be considered a small effect size, $\mathrm{d}=0.5$ represents a 'medium' effect size and $d=0.8$ a 'large' effect size [38]. This indicates if the mean difference between the two groups is less than 0.2 , the effect size is trivial even though the OR is significant. The OR of 1.68, 3.47, and 6.71, are equivalent to Cohen's $d=0.2$ (small), 0.5 (medium) and 0.8 (large) respectively at $1 \%$ level of significance. At 5\% level, the corresponding reference points are 1.52, 2.74, and 4.72. At 10\% level, corresponding reference points are $1.46,2.49$, and 4.14, respectively. The transformation of OR is done, according to Cohen [39].

\section{Results \\ Bivariate analysis results}

Table 1 presents the prevalence of moderate and severe childhood stunting in percentages at various levels of covariates, the level of significance of each variable with that of the response variable set at $P$-value $<0.05$. A litthe more than a quarter $(27.4 \%)$ of children are moderately stunted and one-tenth (10.4\%) is severely stunted in Punjab, Pakistan. Furthermore, $31.5 \%$ of children are moderately stunted between the age group of 36 months to 59 months, whereas about $11.5 \%$ are severely stunted in the same age group. The figure for $0-11$ months is $15 \%$ t moderately stunted, while $5.5 \%$ severely stunted children. Thus, child age is an important and significant predictor of health outcomes. Childbirth order is a significant risk factor for child stunting outcomes. For example, if a child birth order is 3 or above, the child has more likelihood of being stunted (30\%) and severely stunted $(11 \%)$. Whereas a little more than one-fourth (27\%) of first-born are likely to be stunted and one-tenth (10\%) likely to be severely stunted. Of note, $27 \%$ of boys and $28 \%$ of girls are moderately stunted, whereas $10 \%$ of boys and $11 \%$ of girls are severely stunted. Girls are facing significantly higher burden of moderate and severe stunting. Child size at birth is also an important predictor; for example, children born larger than average size are (21\%) moderately stunted and (8\%) severely 
Table 1 Prevalence of moderate and severe childhood stunting at various levels of independent variables in Punjab, Pakistan (Punjab MICS-2014) $(n=25,066)$

\begin{tabular}{|c|c|c|c|c|c|c|}
\hline \multirow[t]{2}{*}{ Variables } & \multicolumn{3}{|c|}{ Stunting (Moderate SD $<-2$ ) } & \multicolumn{3}{|c|}{ Stunting (Severe SD <-3) } \\
\hline & $\overline{\text { Total }}$ & $\begin{array}{l}\text { Yes } \\
\text { N (\%) }\end{array}$ & $\begin{array}{l}\text { No } \\
\text { N (\%) }\end{array}$ & Total & $\begin{array}{l}\text { Yes } \\
\text { N (\%) }\end{array}$ & $\begin{array}{l}\text { No } \\
\text { N (\%) }\end{array}$ \\
\hline Total (\%) & 25,066 & $6865(27.4)$ & $18,201(72.6)$ & 25,066 & $2602(10.4)$ & $22,464(89.6)$ \\
\hline \multicolumn{7}{|c|}{ Individual and Household Level characteristics } \\
\hline Age Group & $<0.001$ & & & $<0.001$ & & \\
\hline $0-5$ months & 2177 & $247(11)$ & $1930(89)$ & 2177 & $99(5)$ & $2078(95)$ \\
\hline $6-11$ & 2767 & $531(19)$ & $2236(81)$ & 2767 & $157(6)$ & $2609(94)$ \\
\hline $12-23$ & 4827 & $1484(31)$ & $3343(69)$ & 4827 & $569(12)$ & $4258(88)$ \\
\hline $24-35$ & 4838 & $1266(26)$ & $3572(74)$ & 4838 & $478(10)$ & $4360(90)$ \\
\hline $36-47$ & 5373 & $1785(33)$ & $3588(76)$ & 5373 & $657(12)$ & $4716(88)$ \\
\hline $48-59$ & 5082 & $1549(30)$ & $3533(70)$ & 5082 & $641(13)$ & 4441 (87) \\
\hline Birth Order & 0.04 & & & 0.023 & & \\
\hline $1 \mathrm{st}$ & 16,391 & 4415 (27) & $11,976(73)$ & 16,435 & $1641(10)$ & $14,794(90)$ \\
\hline 2nd & 7323 & $2046(28)$ & $5277(68)$ & 7323 & $816(11)$ & 6507 (89) \\
\hline $3+$ & 1351 & $403(30)$ & $948(70)$ & 1351 & $144(11)$ & 1207 (89) \\
\hline Gender of Child & 0.024 & & & $<0.001$ & & \\
\hline Boy & 12,712 & $3384(27)$ & 9328 (73) & 12,712 & $1231(10)$ & $11,481(90)$ \\
\hline Girl & 12,354 & $3481(28)$ & $8873(68)$ & 12,354 & $1371(11)$ & 10,983 (89) \\
\hline Size at Birth & 0.001 & & & 0.001 & & \\
\hline Large & 1052 & $218(21)$ & $834(79)$ & 1052 & $80(8)$ & $972(92)$ \\
\hline Average & 8862 & $2281(26)$ & $6581(74)$ & 8862 & $846(10)$ & $8016(90)$ \\
\hline Small & 2633 & $938(36)$ & $1695(64)$ & 2633 & $406(15)$ & $2227(85)$ \\
\hline Diarrhea & $<0.001$ & & & $<0.001$ & & \\
\hline Yes & 4391 & $1344(31)$ & 3047 (69) & 4391 & $556(13)$ & 3835 (87) \\
\hline No & 20,676 & $5522(27)$ & $15,154(73)$ & 20,675 & $2046(10)$ & $18,629(90)$ \\
\hline Breast Feeding & $<0.001$ & & & $<0.001$ & & \\
\hline Yes & 13,950 & 3365 (24) & $10,585(76)$ & 13,949 & $1226(9)$ & $12,723(91)$ \\
\hline No & 703 & $179(25)$ & $524(75)$ & 703 & $81(12)$ & $622(88)$ \\
\hline Mother Education & $<0.001$ & & & $<0.001$ & & \\
\hline Noeducation & 12,270 & $4428(36)$ & $7842(64)$ & 12,270 & $1861(15)$ & $10,409(85)$ \\
\hline Primary & 4517 & $1149(25)$ & $3368(75)$ & 4517 & $367(8)$ & $4150(92)$ \\
\hline Middle & 2481 & $508(20)$ & $1973(80)$ & 2481 & $156(6)$ & $2325(94)$ \\
\hline Secondary & 3101 & $529(17)$ & $2572(83)$ & 3101 & $156(5)$ & $2945(95)$ \\
\hline Higher & 2696 & $249(9)$ & 2447 (91) & 2696 & $63(2)$ & $2633(98)$ \\
\hline Mother Age & 0.042 & & & 0.305 & & \\
\hline$<18$ & 1696 & $499(29)$ & $1197(71)$ & 1696 & $185(11)$ & $1511(89)$ \\
\hline $18-24$ & 5148 & $1323(26)$ & $3825(74)$ & 5148 & $495(10)$ & $4653(90)$ \\
\hline $25-35$ & 13,893 & $3810(27)$ & 10,083 (73) & 13,893 & $1428(10)$ & $12,465(90)$ \\
\hline $36+$ & 4327 & $1232(28)$ & 3095 (72) & 4327 & $492(11)$ & 3835 (89) \\
\hline Antenatal Care & $<0.001$ & & & $<0.001$ & & \\
\hline $1-4$ visits & 6169 & $1725(28)$ & $4444(72)$ & 6169 & $625(10)$ & $5544(90)$ \\
\hline $5+$ visits & 3914 & 718 (18) & $3196(82)$ & 3914 & $253(6)$ & 3661 (94) \\
\hline
\end{tabular}


Table 1 Prevalence of moderate and severe childhood stunting at various levels of independent variables in Punjab, Pakistan (Punjab MICS-2014) $(n=25,066)$ (Continued)

\begin{tabular}{|c|c|c|c|c|c|c|}
\hline \multirow[t]{2}{*}{ Variables } & \multicolumn{3}{|c|}{ Stunting (Moderate SD $<-2$ ) } & \multicolumn{3}{|c|}{ Stunting (Severe SD <-3) } \\
\hline & Total & $\begin{array}{l}\text { Yes } \\
\text { N (\%) }\end{array}$ & $\begin{array}{l}\text { No } \\
\text { N (\%) }\end{array}$ & Total & $\begin{array}{l}\text { Yes } \\
\text { N (\%) }\end{array}$ & $\begin{array}{l}\text { No } \\
\text { N (\%) }\end{array}$ \\
\hline Total (\%) & 25,066 & $6865(27.4)$ & $18,201(72.6)$ & 25,066 & $2602(10.4)$ & $22,464(89.6)$ \\
\hline Postnatal Care & 0.723 & & & 0.431 & & \\
\hline Yes & 4764 & $1315(28)$ & $3449(72)$ & 4764 & $517(11)$ & $4247(89)$ \\
\hline No & 7703 & $2100(27)$ & $5603(73)$ & 7703 & $799(10)$ & $904(90)$ \\
\hline Child Delivery & $<0.001$ & & & $<0.001$ & & \\
\hline Home & 5452 & $1884(35)$ & $3568(65)$ & 5452 & $773(14)$ & $4679(86)$ \\
\hline Hospitals & 7150 & $1567(22)$ & $5583(78)$ & 7150 & $562(8)$ & $6588(92)$ \\
\hline Father Education & $<0.001$ & & & $<0.001$ & & \\
\hline Noeducation & 7335 & $2814(38)$ & $4521(62)$ & 7335 & $1217(17)$ & $6118(83)$ \\
\hline Primary & 4536 & $1419(31)$ & $3117(69)$ & 4536 & $524(12)$ & $4012(88)$ \\
\hline Middle & 4138 & $1024(25)$ & $3114(75)$ & 4138 & $326(8)$ & 3812 (92) \\
\hline Secondary & 5507 & $1144(21)$ & 4363 (79) & 5507 & $391(7)$ & $5116(93)$ \\
\hline Higher & 3548 & $463(13)$ & 3085 (87) & 3548 & $144(4)$ & 3405 (96) \\
\hline WealthIndex & $<0.001$ & & & $<0.001$ & & \\
\hline Poor & 11,167 & 4155 (37) & 7012 (63) & 11,167 & $1770(16)$ & 9397 (84) \\
\hline Middle & 4915 & $1218(25)$ & 3697 (75) & 4915 & $395(8)$ & $4520(92)$ \\
\hline Rich & 8983 & $1491(17)$ & $7492(83)$ & 8983 & $436(5)$ & 8547 (95) \\
\hline Household Size & $<0.001$ & & & $<0.002$ & & \\
\hline 3-4 members & 2873 & $705(25)$ & $2168(75)$ & 2873 & $250(9)$ & $2623(91)$ \\
\hline 5-6 members & 7179 & $1983(28)$ & $5196(68)$ & 7179 & $774(11)$ & 6405 (89) \\
\hline 7-8 members & 6177 & $1838(30)$ & $4339(70)$ & 6177 & $706(11)$ & 5471 (89) \\
\hline $8+$ members & 8836 & $2339(26)$ & $6497(74)$ & 8836 & $871(10)$ & 7965 (90) \\
\hline \multicolumn{7}{|c|}{ Community Level Characteristics } \\
\hline Ethnicity & $<0.001$ & & & $<0.001$ & & \\
\hline Urdu & 1267 & $243(19)$ & $1024(81)$ & 1267 & $76(6)$ & $1191(94)$ \\
\hline Punjabi & 16,141 & $3967(25)$ & $12,174(75)$ & 16,141 & $1332(8)$ & $14,808(92)$ \\
\hline Saraikai & 6040 & $2139(35)$ & $3901(65)$ & 6040 & $973(16)$ & $5067(84)$ \\
\hline Others & 1617 & $515(32)$ & $1102(68)$ & 1617 & $221(13)$ & $1396(87)$ \\
\hline Region & $<0.001$ & & & $<0.001$ & & \\
\hline Urban & 7842 & $1620(21)$ & $6222(79)$ & 7842 & $538(7)$ & $7304(93)$ \\
\hline Rural & 17,224 & $5245(30)$ & $11,979(70)$ & 17,224 & $2063(12)$ & $15,161(88)$ \\
\hline Sanitation & $<0.001$ & & & $<0.001$ & & \\
\hline Improved & 17,903 & $4185(23)$ & $13,718(77)$ & 17,903 & $1429(8)$ & $16,474(92)$ \\
\hline Unimproved & 7163 & $2679(37)$ & $4484(63)$ & 7163 & $1173(16)$ & $5990(84)$ \\
\hline WaterFacilities & $<0.001$ & & & $<0.001$ & & \\
\hline Improved & 23,613 & $6584(28)$ & $17,029(72)$ & 23,613 & $2507(11)$ & $21,106(89)$ \\
\hline Unimproved & 1453 & $281(19)$ & $1172(81)$ & 1453 & $94(7)$ & $1359(93)$ \\
\hline Water Treatment & $<0.001$ & & & $<0.001$ & & \\
\hline Treated & 1524 & $228(15)$ & $1296(85)$ & 1425 & $82(5)$ & $1443(95)$ \\
\hline Untreated & 23,542 & $6638(28)$ & $16,904(72)$ & 23,524 & $2521(11)$ & $21,023(89)$ \\
\hline
\end{tabular}


Table 1 Prevalence of moderate and severe childhood stunting at various levels of independent variables in Punjab, Pakistan (Punjab MICS-2014) $(n=25,066)$ (Continued)

\begin{tabular}{|c|c|c|c|c|c|c|}
\hline \multirow[t]{2}{*}{ Variables } & \multicolumn{3}{|c|}{ Stunting (Moderate SD $<-2$ ) } & \multicolumn{3}{|c|}{ Stunting (Severe SD <-3) } \\
\hline & Total & $\begin{array}{l}\text { Yes } \\
\text { N (\%) }\end{array}$ & $\begin{array}{l}\text { No } \\
\text { N (\%) }\end{array}$ & Total & $\begin{array}{l}\text { Yes } \\
\text { N (\%) }\end{array}$ & $\begin{array}{l}\text { No } \\
\text { N (\%) }\end{array}$ \\
\hline Total (\%) & 25,066 & $6865(27.4)$ & $18,201(72.6)$ & 25,066 & $2602(10.4)$ & $22,464(89.6)$ \\
\hline \multicolumn{7}{|l|}{ Regional LevelCharacteristics } \\
\hline Food InsecurityIndex (FII) & $<0.001$ & & & $<0.001$ & & \\
\hline Insecure & 2662 & 1035 (39) & $1626(61)$ & 2662 & $482(18)$ & $2179(82)$ \\
\hline Vulnerable & 8875 & $2598(29)$ & $6277(71)$ & 8875 & $1016(11)$ & 7859 (89) \\
\hline Secure & 13,529 & $3232(24)$ & $10,297(76)$ & 13,529 & $1104(8)$ & $12,425(92)$ \\
\hline Division & $<0.001$ & & & $<0.001$ & & \\
\hline Rawalpindi & 1909 & $312(16)$ & $1597(84)$ & 1909 & $86(5)$ & $1823(95)$ \\
\hline Bahawalpur & 2930 & $1016(35)$ & $1914(65)$ & 2930 & $432(15)$ & $2498(85)$ \\
\hline D.G Khan & 2822 & $1112(39)$ & $1710(61)$ & 2822 & $528(19)$ & $2294(81)$ \\
\hline Faisalabad & 3115 & $758(24)$ & $2357(76)$ & 3115 & $272(9)$ & $2843(91)$ \\
\hline Gujranwala & 3234 & $727(22)$ & 2507 (78) & 3234 & $235(7)$ & 2999 (93) \\
\hline Lahore & 4416 & $1093(25)$ & $3323(75)$ & 4416 & 378 (9) & 4038 (91) \\
\hline Multan & 2853 & $823(29)$ & $2030(71)$ & 2853 & $326(11)$ & 2527 (89) \\
\hline Sahiwal & 1880 & $522(28)$ & $1358(71)$ & 1880 & $188(10)$ & $1691(90)$ \\
\hline Sargoda & 1904 & $500(26)$ & $1404(74)$ & 1904 & 155 (8) & 1749 (92) \\
\hline
\end{tabular}

stunted, but those born with smaller than average birth size are $36 \%$ moderately and $15 \%$ severely stunted. Almost $24 \%$ of children who breastfeed are moderately and about $9 \%$ severely stunted compared to $25 \%$; those who are not breast-feed are moderately stunted and $12 \%$ severely stunted. Children having diarrhea are more likely to be stunted (31\%) compared to those who are not having diarrhea (27\%).

Lower the mother's education higher the chances that a child is facing stunting. For example, a woman with low education has a higher likelihood of having stunted children (36\% moderate and 15\% severe) compared to highly educated ( $9 \%$ moderately stunted and $2 \%$ severely stunted). Children whose father is educated (13\% moderate and $4 \%$ severe stunting) are less likely to be stunted compared to those children whose father is not educated (38\% moderate and $17 \%$ severe stunting).

If a woman marries at age less than 18 years as compared to those marries after 36 years (29\% of their children are moderately stunted and $11 \%$ severely stunted) is more likely to a borne stunted child. A significantly high number of children 30\% living in rural areas are moderately stunted relative to $21 \%$ in urban areas, whereas about $12 \%$ severely stunted compared to $7 \%$ in urban areas. There are significant differences among the administrative divisions of the Punjab province. For example, Rawalpindi division has a significantly lower prevalence of moderate and severe stunting (16\% moderate and 5\% severe stunting) compared D. G. Khan,
Bahawalpur and Multan (39, 35 and 29\% moderate stunting and 19, 15 and $11 \%$ severe stunting) respectively. Every fourth child 25\%, (1.3-2.7) in Lahore, the provincial capital of Punjab, is moderately stunted and every tenth child $9 \%(1.3-2.7)$, is severely stunted. Those households having unimproved sanitation facilities are highly likely to have more stunted children (sanitation: $37 \%$ versus $23 \%$ ). Surprisingly, with improved water facilities, the likelihood of stunting increases (19\% unimproved versus improved 28\%). However, with unimproved water facility the sample households are less than 5\%. Almost 39\% (18\%) of those children living in food insecure districts are moderately (severely) stunted relative to $29 \%(1 \%)$ in vulnerable (borderline) districts and $24 \%(8 \%)$ in food secure districts.

\section{Multivariate analysis results}

Tables 2 and 3 present the results of the multilevel logistic regression analyses and the results are presented as Odds ratio (OR) with their 95\% Confidence Interval (95\% CI). The $L R$ test favored the multilevel logistic regression instead of conventional logistic regression, shown by the statistically significant $\left(L R X^{2}=1005.94\right.$, $P<0.001)$ for moderate stunting and $\left(L R X^{2}=628.88\right.$, $P<0.001$ ) for severe stunting (see Tables 2 and 3).

Model 2 in Table 2 presents the results of only child specific and parent's level characters and their association with moderate stunting. For example, compared to the reference category of zero to 5 months, children of 6 
Table 2 Multivariable Regression results of Multilevel models for moderate stunting, MICS Punjab-2014 $(n=25,066)$

Moderate Stunting SD $>-2$
Variables Model $1^{\text {a }}$
Individual and Household Level Characteristics
Child age group (Reference Category 0-5 months,
6-11
$\begin{aligned} & 12-23 \\ & 24-35 \\ & 36-47 \\ & 48-59\end{aligned}$

Child Birth order (Reference Category First Born)

2

$3+$

Child Gender (Reference Category Boy)

Girl

Child size at birth (Reference Category Small Size)

$$
\text { Average }
$$

Large

Diarrhea (Reference Category No)

$$
\text { Yes }
$$

Breast Feeding (Reference Category Yes)

No

$$
\begin{aligned}
& (0.52) 2.58^{* * *}[1.9,3.5] \\
& (1.09) 7.25^{* * *}[5.1,10.3] \\
& (1.01) 6.25^{* * *}[4.1,9.5] \\
& (1.50) 15.36^{* * *}[7.3,32.5] \\
& (1.42) 13.28^{* * *}[6.5,27.2]
\end{aligned}
$$

(0.25) $1.57^{* * *}[1.4,1.8]$

(0.63) $3.14^{* * *}[2.4,4.1]$

$(0.07) 1.14^{* * *}[1.1,1.2]$

(0.27) $0.61^{* * *}[0.45,0.81]$

$(0.24) 0.65^{* * *}[0.56,0.75]$

(0.17) $0.73^{* * *}[0.6,0.8]$

(0.15) $1.35 *[1.0,1.7]$

Mother Education (Reference Category No Education)

$\begin{array}{ll}\text { Primary } & (0.22) 0.67^{* * *}[0.6,0.8] \\ \text { Middle } & (0.37) 0.51^{* * *}[0.4,0.6] \\ \text { Secondary } & (0.42) 0.47^{* * *}[0.4,0.6] \\ \text { Higher } & (0.76) 0.25^{* * *}[0.2,0.3]\end{array}$

Mother Age (Reference Category age of women < 18 years of age)

$$
\begin{aligned}
& 18-24 \\
& 25-35 \\
& 36-49
\end{aligned}
$$

Postnatal Care (Reference Category Yes) No

$$
\begin{aligned}
& (0.03) 0.94[0.7,1.2] \\
& (0.04) 0.93[0.8,1.1] \\
& (0.08) 0.86[0.7,1.0]
\end{aligned}
$$

$(0.02) 0.97[0.9,1.1]$

$(0.07) 0.88[0.8,1.0]$

(0.04) $0.93[0.8,1.1]$

(0.09) $0.85 *[0.7,1.0]$

(0.24) $0.65^{* * *}[0.6,0.7]$

(0.32) $0.56^{* * *}[0.5,0.6]$

(0.50) $0.40^{* * *}[0.3,0.5]$

Father Education (Reference category No Education)

Primary
Middle
Secondary
Higher

Wealth index (Reference category Poor)

Middle

Rich
Model $3^{c}$ Cohen's $d \&$ OR [95\% Cl]
Model $4{ }^{d}$ Cohen's $d$ \& OR [95\% Cl]

$(0.53) 2.61^{* * *}[1.9,3.5]$
$(1.10) 7.41^{* * *}[5.2,10.5]$
$(1.01) 6.26^{* * *}[4.1,9.5]$
$(1.51) 15.54^{* * *}[7.3,33.1]$
$(1.42) 13.33^{* * *}[6.4,27.6]$

(0.24) $1.55^{* * *}[1.4,1.7]$

(0.62) $3.05^{* * *}[2.3,4.0]$

(0.62) $3.06^{* * *}[2.3,4.0]$

$(0.07) 1.14^{* *}[1.1,1.2]$

$(0.07) 1.14^{* * *}[1.1,1.2]$

(0.27) $0.60^{* * *}[0.46,0.80]$

(0.27) $0.61^{* * *}[0.46,0.81]$

$0.24) 0.65^{* * *}[0.55,0.76]$

(0.16) $0.74^{* * *}[0.7,0.8]$

(0.15) $0.75^{* * *}[0.7,0.9]$

(0.15) $1.36 *[1.1,1.8]$

(0.15) $1.35^{*}[1.0,1.8]$

(0.14) $0.77^{* * *}[0.7,0.9]$

(0.14) $0.77^{* * *}[0.7,0.9]$

(0.25) $0.63^{* * *}[0.5,0.8]$

$(0.25) 0.64^{* * *}[0.5,0.8]$

(0.24) $0.62^{* * *}[0.5,0.8]$

(0.24) $0.63^{* * *}[0.5,0.8]$

(o.59) $0.34^{* * *}[0.3,0.4]$

(0.59) $0.34^{* * *}[0.3,0.4]$

(0.005) $0.99[0.8,1.2]$

(0.005) $0.99[0.8,1.3]$

(0.02) $0.97[0.8,1.2]$

(0.02) $0.97[0.8,1.2]$

(0.07) $0.88[0.7,1.1]$

$0.07) 0.88[0.7,1.1]$

(0.02) $0.98[0.9,1.1]$

(0.02) $0.97[0.9,1.1]$

(0.03) $0.94[0.8,1.1]$

(0.03) $0.95[0.8,1.1]$

$(0.01) 0.98[0.8,1.1]$

(0.01) $0.98[0.8,1.1]$

(0.06) $0.90[0.8,1.0]$

(0.06) $0.91[0.8,1.0]$

(0.18) $0.71^{* * *}[0.6,0.8]$

(0.18) $0.72^{* * *}[0.6,0.8]$

$(0.23) 0.64^{* * *}[0.5,0.7]$

(0.24) $0.65^{* * *}[0.6,0.8]$

(0.40) $0.48^{* * *}[0.4,0.6]$

(0.40) $0.48^{* * *}[0.4,0.6]$

(0.23) $0.64^{* * *}[0.7,0.8]$

$(0.24) 0.65^{* * *}[0.6,0.8]$

(0.41) $0.47^{* * *}[0.5,0.6]$ 
Table 2 Multivariable Regression results of Multilevel models for moderate stunting, MICS Punjab-2014 $(n=25,066)(C o n t i n u e d)$

\begin{tabular}{|c|c|c|c|c|}
\hline \multicolumn{5}{|l|}{ Moderate Stunting SD $>-2$} \\
\hline Variables & Model $1^{\text {a }}$ & $\begin{array}{l}\text { Model 2 } 2^{\mathrm{b}} \text { Cohen's d \& OR } \\
{[95 \% \mathrm{Cl}]}\end{array}$ & $\begin{array}{l}\text { Model 3c Cohen's d \& OR } \\
{[95 \% \mathrm{Cl}]}\end{array}$ & $\begin{array}{l}\text { Model } 4^{d} \text { Cohen's d \& OR } \\
{[95 \% \mathrm{Cl}]}\end{array}$ \\
\hline \multicolumn{5}{|c|}{ HH size (Reference category HH size 3-4 members) } \\
\hline $5-6$ members & & & $(0.05) 1.11[1.0,1.4]$ & $(0.05) 1.10[1.0,1.3]$ \\
\hline 7-8 members & & & $(0.11) 1.23^{*}[1.0,1.5]$ & $(0.11) 1.22^{*}[1.0,1.4]$ \\
\hline$>8$ members & & & $(0.09) 1.19[1.0,1.5]$ & $(0.08) 1.17[1.0,1.4]$ \\
\hline \multicolumn{5}{|c|}{ Community Level Characteristics } \\
\hline \multicolumn{5}{|c|}{ Ethnicity (Refernececategory Urdu) } \\
\hline Punjabi & & & $(0.08) 0.86[0.7,1.1]$ & $(0.08) 0.87[0.7,1.1]$ \\
\hline Saraikai & & & $(0.03) 0.95[0.7,1.2]$ & $(0.06) 0.90[0.7,1.2]$ \\
\hline Others & & & $(0.04) 1.08[0.8,1.4]$ & $(0.04) 1.08[0.8,1.4]$ \\
\hline \multicolumn{5}{|c|}{ Region (Reference category Urban) } \\
\hline Rural & & $(0.11) 1.22^{* * *}[1.1,1.3]$ & $(0.03) 0.93[0.9,1.0]$ & $(0.03) 0.94[0.9,1.0]$ \\
\hline \multicolumn{5}{|c|}{ Sanitation facility (Reference category Unimproved) } \\
\hline Improved & & & $(0.08) 0.86^{*}[0.7,1.0]$ & $(0.08) 0.87^{*}[0.8,1.0]$ \\
\hline \multicolumn{5}{|c|}{ Drinking water facility (Reference category Unimproved) } \\
\hline Improved & & & $(0.07) 1.13[0.7,1.5]$ & $(0.07) 1.12[0.8,1.5]$ \\
\hline \multicolumn{5}{|c|}{ Water treatment (Reference category Treated) } \\
\hline Untreated & & & $(0.13) 1.26[1.0,1.7]$ & $(0.13) 1.25[1.0,1.6]$ \\
\hline \multicolumn{5}{|c|}{ Regional LevelCharacteristics } \\
\hline \multicolumn{5}{|c|}{ Food Insecurity index (Reference category Food Insecure) } \\
\hline Food Vulnerable & & & & $(0.14) 0.77^{*}[0.6,0.9]$ \\
\hline Food Secure & & & & $(0.19) 0.71^{* * *}[0.6,0.9]$ \\
\hline \multicolumn{5}{|c|}{ Division (Reference category Rawalpindi Division) } \\
\hline Bahawalpur & & & & $(0.24) 1.56^{*}[1.1,2.2]$ \\
\hline D.G Khan & & & & $(0.23) 1.51^{*}[1.0,2.2]$ \\
\hline Faisalabad & & & & $(0.20) 1.45^{* *}[1.1,1.9]$ \\
\hline Gujranwala & & & & $(0.19) 1.41^{*}[1.1,1.9]$ \\
\hline Lahore & & & & $(0.35) 1.88^{* * *}[1.3,2.7]$ \\
\hline Multan & & & & $(0.19) 1.41^{*}[1.1,1.9]$ \\
\hline Sahiwal & & & & $(0.18) 1.39 *[1.0,1.9]$ \\
\hline Sargodha & & & & $(0.07) 1.13[0.8,1.6]$ \\
\hline Constant & $-1.43(0.13)^{* * *}$ & $0.038(0.02)^{* * *}$ & $0.040(0.019)^{* * *}$ & $0.030(0.010)^{* * *}$ \\
\hline Level-1 (Household) & $1.18(0.10)^{* * *}$ & $2.19 * * *(1.2,3.7)$ & $2.17^{* * *}(1.3,3.7)$ & $2.18^{* * *}(1.3,3.7)$ \\
\hline Level-2 (Community) & $0.53(0,03)^{* * *}$ & $0.15^{* * *}(0.08,0.3)$ & $\left.0.14^{* * *} 0.07,0.3\right)$ & $0.14^{* * *}(0.07,0.3)$ \\
\hline Level-3 (District) & $0.37(0.03)^{* * *}$ & $0.04 * * *(0.02,0.1)$ & $0.03^{* * *}(0.01,0.08)$ & $0.004(0.0,0.02)$ \\
\hline Chi2 & $(1005.94)^{* * *}$ & & & \\
\hline
\end{tabular}

Cohen's $d$ values are on the left side of each column in parenthesis

$O R=$ Odds Ratio, $C l=95 \%$ Confidence Intervals in brackets. ${ }^{*} p<0.05, * * 0<0.01, * * * 0.001$

$a=$ base model (unconditional three level hierarchical model), $b=$ hierarchical model with child and parental characteristics, $c=$ hierarchical model with child, parental and household characteristics and $d=$ hierarchical model with child, parental, household and districts food insecurity index and division

to 11 months are 2 and half times more likely to be stunted $(\mathrm{OR}=2.58, \mathrm{CI}=1.9-3.5)$, whereas children of age 12-23 months are 7 times more prone to be stunted $(\mathrm{OR}=7.25$ (5.1,10.3). Girls are significantly more likely to be stunted than boys $(\mathrm{OR}=1.14,95 \% \mathrm{CI}=1.1-1.2)$.
Children whose birth order is second is significantly likely to be stunted $(\mathrm{OR}=1.57 \quad 95 \% \quad \mathrm{CI}=1.4-1.8)$ whereas children whose birth order is 3 or more is 3 times more likely to be stunted $(\mathrm{OR}=3.1495 \% \mathrm{CI}=$ 2.4-4.1). Children with no breastfeeding are not 
Table 3 Multivariable Regression results of Multilevel models for severe stunting, MICS Punjab-2014 $(n=25,066)$

\begin{tabular}{|c|c|c|}
\hline \multicolumn{3}{|c|}{ Severe Stunting SD $>-3$} \\
\hline Variables & Model $1^{\text {a }}$ & $\begin{array}{l}\text { Model 2b Cohen's d \& } \\
{[95 \% \mathrm{Cl}]}\end{array}$ \\
\hline \multicolumn{3}{|c|}{ Individual and Household Level Characteristics } \\
\hline \multicolumn{3}{|c|}{ Child age group (months) (Reference Category 0-5 months) } \\
\hline $06-11$ & & $(0.24) 1.56[1.9,3.5]$ \\
\hline $12-23$ & & $(0.87) 4.85^{* * *}[5.1,10.3]$ \\
\hline $24-35$ & & $(0.84) 4.62^{* * *}[4.1,9.5]$ \\
\hline $36-47$ & & $(1.02) 6.36^{* * *}[7.3,32.5]$ \\
\hline $48-59$ & & $(1.07) 6.98^{* * *}[6.5,27.2]$ \\
\hline
\end{tabular}

Child order (Reference Category First Born)

Second

More than 2

(0.31) $1.76^{* * *}[1.4,1.8]$

$(0.55) 2.71^{* * *}[2.4,4.1]$

Gender of child (Reference Category Boy)

Girl

Child size at birth (Reference Category Small Size)

$$
\text { Average }
$$

Large

$$
(0.13) 1.27^{* * *}[1.1,1.2]
$$

(0.20) $0.69^{* *}[0.5,0.9]$

(0.25) $0.63^{* * *[.4,0.8]}$

(0.25) $0.64^{* * *}[0.6,0.8]$

(0.34) $1.87^{* *}[1.0,1.7]$

(0.28) $0.60^{* * *}[0.6,0.8]$

(0.43) $0.46^{* * *}[0.4,0.6]$

(0.50) $0.40^{* * *}[0.4,0.6]$

(0.83) $0.22^{* * *}[0.2,0.3]$

Higher

Mother Age (years) (Reference Category age of women $<18$ years of age)

$$
\begin{aligned}
& \text { Age }>=18 \&<=24 \\
& \text { Age }>24 \&<=35 \\
& \text { Age }>36
\end{aligned}
$$

(0.005) $0.99[0.7,1.2]$

(0.06) $0.89[0.8,1.1]$

(0.04) $0.92[0.7,1.0]$

Postnatal Care (Reference category yes)

No

$(0.03) 0.95[0.9,1.1]$

Prenatal Care (Reference category 1-4 visits)

More than 4 visit

$(0.005) 0.99[0.8,1.0]$

$(0.03) 0.96[0.8,1.1]$

Hospital

Father education (Reference category No Education)

$\begin{array}{ll}\text { Primary } & (0.12) 0.80^{*}[0.7,1.0] \\ \text { Middle } & (0.32) 0.56^{* * *}[0.6,0.7] \\ \text { Secondary } & (0.32) 0.56^{* * *}[0.5,0.6] \\ \text { Higher } & (0.49) 0.41^{* * *}[0.3,0.5]\end{array}$

Wealth Index (Reference category Poor)

Middle

Rich
Model $3^{c}$ Cohen's $d \&$ OR [95\% Cl]

$$
\begin{aligned}
& (0.24) 1.57[1.0,2.6] \\
& (0.88) 4.92^{* * *}[2.9,8.2] \\
& (0.84) 4.61^{* * *}[2.5,8.7] \\
& (1.02) 6.35^{* * *}[2.3,17.9] \\
& (1.07) 6.97^{* * *}[2.4,20.3]
\end{aligned}
$$

Model $4^{d}$ Cohen's $d$ \& OR [95\% Cl]

$$
\text { (o.30) } 1.73^{* * *}[1.4,2.1]
$$

(0.53) $2.62^{* * *}[1.7,4.0]$

$$
\begin{aligned}
& (0.24) 1.57[1.0,2.6] \\
& (0.88) 4.94^{* * *}[3.0,8.2] \\
& (0.84) 4.61 * * *[2.5,8.7] \\
& (1.02) 6.42^{* * *}[2.3,18.3] \\
& (1.08) 7.04^{* * *}[2.4,20.7]
\end{aligned}
$$

$$
(0.30) 1.72^{* * *}[1.4,2.1]
$$$$
\text { (0.53) } 2.60^{* * *}[1.7,3.9]
$$

(0.13) $1.27^{* * *}[1.2,1.4]$

(0.13) $1.27^{* * *}[1.2,1.4]$

(0.20) $0.68^{* *}[0.5,0.9]$

$(0.20) 0.70^{* *}[0.5,0.9]$

(0.27) $0.61^{* * *[.4,8]}$

$(0.26) 0.62^{* * *}[0.4,0.8]$

(0.25) $0.65^{* * *}[0.6,0.8]$

$(0.25) 0.65^{* * *}[0.6,0.8]$

$\left(0.3401 .89^{* *}[1.3,2.8]\right.$

$\left(0.3401 .87^{* *}[1.3,2.8]\right.$

(0.18) $0.72^{* *}[0.6,0.9]$

(0.18) $0.72 * *[0.6,0.9]$

(0.28) $0.61^{* * *}[0.5,0.8]$

(0.28) $0.61^{* * *}[0.5,0.8]$

(0.32) $0.56^{* * *}[0.4,0.7]$

(0.32) $0.56^{* * *}[0.4,0.7]$

(0.64) $0.31^{* * *}[0.2,0.5]$

(0.64) $0.31^{* * *}[0.2,0.5]$

(0.02) $1.03[0.8,1.3]$

(0.04) $0.93[0.7,1.2]$

(0.04) $0.93[0.7,1.2]$

(0.02) $1.03[0.8,1.3]$

(0.04) $0.93[0.7,1.2]$

(0.04) $0.93[0.7,1.2]$

(0.03) $0.95[0.8,1.2]$

(0.03) $0.94[0.8,1.2]$

(0.03) $1.06[0.9,1.3]$

(0.03) $1.07[0.9,1.3]$

(0.02) $1.02[0.8,1.3]$

(0.02) $1.03[0.8,1.3]$

(0.08) $0.86[0.7,1.0]$

(0.08) $0.87[0.7,1.0]$

(0.26) $0.63^{* * *}[0.5,0.7]$

$(0.25) 0.64^{* * *}[0.6,0.8]$

$(0.23) 0.66^{* * *}[0.5,0.8]$

$(0.23) 0.67^{* *}[0.5,0.9]$

(0.38) $0.50^{* * *}[0.4,0.6]$

$(0.37) 0.51^{* * *}[0.4,0.6]$

$(0.27) 0.61^{* * *}[0.5,0.7]$

$(0.27) 0.61^{* * *}[0.5,0.7]$

(0.43) $0.46^{* * *}[0.4,0.6]$ 
Table 3 Multivariable Regression results of Multilevel models for severe stunting, MICS Punjab-2014 $(n=25,066)($ Continued)

\begin{tabular}{|c|c|c|c|c|}
\hline \multicolumn{5}{|l|}{ Severe Stunting SD $>-3$} \\
\hline Variables & Model $1^{a}$ & $\begin{array}{l}\text { Model 2b Cohen's d \& OR } \\
{[95 \% \mathrm{Cl}]}\end{array}$ & $\begin{array}{l}\text { Model } 3^{c} \text { Cohen's d \& OR } \\
{[95 \% \mathrm{Cl}]}\end{array}$ & $\begin{array}{l}\text { Model } 4^{d} \text { Cohen's d \& OR } \\
{[95 \% \mathrm{Cl}]}\end{array}$ \\
\hline \multicolumn{5}{|c|}{ HH size (Reference category HH size 3-4 members) } \\
\hline 5-6 members & & & $(0.09) 1.18[0.9,1.5]$ & $(0.09) 1.18[0.9,1.5]$ \\
\hline 7-8 members & & & $(0.11) 1.23[0.9,1.6]$ & $(0.11) 1.23[0.9,1.6]$ \\
\hline$>8$ members & & & $(0.11) 1.23[1.0,1.6]$ & $(0.11) 1.23[1.0,1.6]$ \\
\hline \multicolumn{5}{|c|}{ Community Level Characteristics } \\
\hline \multicolumn{5}{|c|}{ Ethnicity (Reference category Urdu) } \\
\hline Punjabi & & & $(0.09) 0.85[0.5,1.3]$ & $(0.09) 0.85[0.5,1.3]$ \\
\hline Saraikai & & & $(0.07) 1.15[0.8,1.7]$ & $(0.06) 1.11[0.7,1.7]$ \\
\hline Others & & & $(0.08) 1.17[0.8,1.8]$ & $(0.09) 1.18[0.8,1.8]$ \\
\hline \multicolumn{5}{|c|}{ Region (Reference category Urban) } \\
\hline Rural & & $(0.09) 1.18[1.0,1.4]$ & $(0.09) 0.85[0.7,1.0]$ & $(0.09) 0.87[0.7,1.0]$ \\
\hline \multicolumn{5}{|c|}{ Sanitation facility (Reference category Unimproved) } \\
\hline Improved & & & $(0.13) 0.79^{* *}[0.7,0.9]$ & $(0.13) 0.79^{* *}[0.7,0.9]$ \\
\hline \multicolumn{5}{|c|}{ Drinking water facility (Reference category Unimproved) } \\
\hline Improved & & & $(0.08) 1.17[0.9,1.6]$ & $(0.07) 1.15[0.9,1.5]$ \\
\hline \multicolumn{5}{|c|}{ Water treatment (Reference category Treated) } \\
\hline Untreated & & & $(0.07) 1.15[0.7,1.8]$ & $(0.07) 1.17[0.8,1.8]$ \\
\hline \multicolumn{5}{|c|}{ Regional Level Characteristics } \\
\hline \multicolumn{5}{|c|}{ Food Insecurity index (Reference category Food Insecure) } \\
\hline Food Vulnerable & & & & $(0.08) 0.87[0.6,1.2]$ \\
\hline Food Secure & & & & $(0.14) 0.77[0.6,1.1]$ \\
\hline \multicolumn{5}{|c|}{ Division (Reference category Rawalpindi Division) } \\
\hline Bahawalpur & & & & $(0.25) 1.57^{*}[1.0,2.4]$ \\
\hline D.G Khan & & & & $(0.34) 1.84^{* *}[1.2,2.9]$ \\
\hline Faisalabad & & & & $(0.27) 1.63^{*}[1.1,2.4]$ \\
\hline Gujranwala & & & & $(0.26) 1.60^{*}[1.0,2.5]$ \\
\hline Lahore & & & & $(0.38) 1.98^{* *}[1.2,3.2]$ \\
\hline Multan & & & & $(0.19) 1.41[0.9,2.1]$ \\
\hline Sahiwal & & & & $(0.25) 1.57^{*}[1.1,2.3]$ \\
\hline Sargodha & & & & $0.93[0.6,1.4]$ \\
\hline Constant & $-3.14(0.25) * * *$ & $0.011(0.005)^{* * *}$ & $0.013(0.010)^{* * *}$ & $0.012(0.001)^{* * *}$ \\
\hline Level-1 (Household) & $1.16(0.16)^{* * *}$ & $2.51 * * *(1.3,4.7)$ & $2.50^{* * *}(1.3,3.7)$ & $2.50^{* * *}(1.3,4.7)$ \\
\hline Level - 2 (community) & $0.55(0.05)^{* * *}$ & $0.20 * *(0.1,0.4)$ & $0.19^{* * *}(0.07,0.3)$ & $0.19^{* * *}(0.09 .0 .4)$ \\
\hline Level - 3 (District) & $0.48(0.06)^{* * *}$ & $0.10 * * *(0.04,0.2)$ & $0.06^{* * *}(0.01,0.08)$ & $0.02^{*}(0.0,0.05)$ \\
\hline Chi2 & $(628.88)^{* * *}$ & & & \\
\hline
\end{tabular}

Cohen's $d$ values are on the left side of each column in parenthesis

OR Odds Ratio, $\mathrm{Cl}=95 \%$ Confidence Intervals in brackets. ${ }^{*} p<0.05, * * 0<0.01, * * * 00.001$

$a=$ base model (unconditional three level hierarchical model), $b=$ hierarchical model with child and parental characteristics, $c=$ hierarchical model with child, parental and household characteristics and $d=$ hierarchical model with child, parental, household and districts food insecurity index and division

significantly likely to be moderately stunted $(\mathrm{OR}=1.35$ 95\% CI $=1-1.7)$ than those children who had breastfeeding. Children with no diarrhea are significantly less likely to be stunted compared to those having an episode of diarrhea $(\mathrm{OR}=0.7395 \% \mathrm{Cl}=0.6-0.8)$. Children whose size at birth perceived as average compared to small size at birth are less likely to be moderately stunted. (OR = $0.6195 \% \mathrm{CI}=0.45-0.81)$. Mothers' education is a significant covariate of moderate stunting. Compared to no education, reference category, children of highly 
Table 4 Results from random intercept model: Measures of variation Variance Partition Coefficient (VPC) and Inter Class Correlation (ICC)

\begin{tabular}{|c|c|c|c|c|c|c|c|c|}
\hline \multirow{2}{*}{$\begin{array}{l}\text { Models } \\
\text { Standard Deviation }\end{array}$} & \multicolumn{2}{|c|}{ Model 1} & \multicolumn{2}{|c|}{ Model 2} & \multicolumn{2}{|c|}{ Model 3} & \multicolumn{2}{|c|}{ Model 4} \\
\hline & $-2 \mathrm{SD}$ & $-3 \mathrm{SD}$ & $-2 S D$ & $-3 \mathrm{SD}$ & $-2 \mathrm{SD}$ & $-3 \mathrm{SD}$ & $-2 \mathrm{SD}$ & $-3 S D$ \\
\hline \multicolumn{9}{|c|}{ Variance Partition Coefficient (VPC) } \\
\hline$V P C_{h h}=\frac{\sigma_{h h}^{2}}{\sigma_{d i s t}^{2}+\sigma_{\text {comm }}^{2}+\sigma_{h h}^{2}+\pi 2 / 3}$ & 0.22 & 0.21 & 0.37 & 0.41 & 0.38 & 0.40 & 0.38 & 0.42 \\
\hline$V P C_{\text {comm }}=\frac{\sigma_{\text {comm }}^{2 n}}{\sigma_{\text {dist }}^{2}+\sigma_{\text {comm }}^{2}+\sigma_{\text {hh }}^{2}+\pi 2 / 3}$ & 0.10 & 0.10 & 0.03 & 0.03 & 0.04 & 0.03 & 0.03 & 0.03 \\
\hline$V P C_{\text {distt }}=\frac{\sigma_{\text {dist }}^{2}}{\sigma_{\text {dist }}^{2}+\sigma_{\text {comm }}^{2}+\sigma_{\text {hh }}^{2}+\pi 2 / 3}$ & 0.07 & 0.08 & 0.007 & 0.02 & 0.006 & 0.02 & 0.007 & 0.003 \\
\hline \multicolumn{9}{|l|}{ Intra Class Correlation (ICC) } \\
\hline$I C C_{\text {comm }}=\frac{\sigma_{\text {dist }}^{2}+\sigma_{\text {comm }}^{2}}{\sigma_{\text {dist }}^{2}+\sigma_{\text {comm }}^{2}+\sigma_{\text {hh }}^{2}+\pi 2 / 3}$ & 0.17 & 0.19 & 0.03 & 0.05 & 0.03 & 0.05 & 0.03 & 0.03 \\
\hline$I C C_{\text {distt }}=\frac{\sigma_{\text {dist }}^{2}}{\sigma_{\text {dist }}^{2}+\sigma_{\text {comm }}^{2}+\sigma_{\text {hh }}^{2}+\pi 2 / 3}$ & 0.07 & 0.08 & 0.007 & 0.02 & 0.006 & 0.02 & 0.007 & 0.003 \\
\hline
\end{tabular}

Note: total variance $=\sigma_{\text {distt }}^{2}+\sigma_{\text {comm }}^{2}+\sigma_{h h}^{2}+\pi 2 / 3$

educated mothers are significantly less likely to be moderately stunted $(\mathrm{OR}=0.2595 \% \mathrm{CI}=0.2-0.3)$. Whereas children of Primary $(\mathrm{OR}=0.6795 \% \mathrm{CI}=0.6-0.8)$ and Middle school completed mothers $(\mathrm{OR}=0.5195 \% \mathrm{CI}=$ 0.4-0.6) are significantly less likely than no educated mothers to be stunted. Children of a father who has higher education are less likely to be stunted ( $\mathrm{OR}=0.40$ $95 \% \mathrm{CI}=0.3-0.5)$. Living in rural areas is a risk factor of moderate stunting. The results of severe stunting (less than minus $3 \mathrm{SD}$ ) are presented in Table 3.

Table 4 presents the results of $V P C$ and $I C C$ for both moderate and severe stunting. The total variance (unconditional model 1) by combining the variation at three levels (district, community and household) is estimated to be $5.37(=0.37+0.53+1.18+3.29)^{2}$ for moderate stunting. The district level explains $7 \%(=0.37 / 5.37)$ proportional change in variance (VPC), the community level variance shows $10 \%(=0.53 / 5.37)$ of variation and the household level variation implies that the risk of stunting varies significantly by $0.22 \%(=1.18 / 5.37)$. Similarly, looking at the ICC statistics of moderate stunting (unconditional model 1), we find that the district and community level ICC is 0.07 and 0.17 respectively. Thus, the correlation between two households from the same district, but different community is 0.07 , while the correlation between two households from the same district and same community is higher at 0.17 . This means that households in the same community have higher chance of correlation than the households of adjacent communities. This also shows that variance in the odds of a child being stunted is explained $7 \%$ by district and $17 \%$ by community characteristics.

In summary, the $V P C$ and $I C C$ statistics show a high degree of clustering in the Punjab stunting data. However, the majority of the variation in stunting both

\footnotetext{
${ }^{2}$ In the latent variable method, the individual variance level is $\pi 2 / 3$, that approximately is 3.29 . That is why we have added it to the total variance.
}

moderate and severe lies at the household level. The differences could be due to income inequality, access to the health care facilities, food insecurity of households. Hence, the findings imply that stark variation exists among household demographic and socio-economic characteristics. Therefore, for policy relevance, household level nutrition related policy should be prioritized, followed by community and districts level measures.

\section{Discussion}

We have analyzed the determinants of under nutrition (stunting) in Punjab Pakistan, which include 25,066 children of less than 5 years using multilevel hierarchical models. Our results indicate that stunting and severe stunting is a daunting public health challenge of the province of Punjab, Pakistan, because almost a little more than one fourth $(27.4 \%)$ of children are moderately stunted and $10 \%$ are severely stunted in 2014 . This number is alarming. The key individual and household level covariates significantly associated with stunting and severe stunting are; age of the child, birth order of the child, being female, being smaller in size at birth, episode of diarrhea, breast feeding, mother and father education, and wealth status of the household. A number of studies done on Africa and South Asia found that the risk of stunting and severe stunting increases with an increase in the age of a child [21, 29, 40-42]. It is because stunting can start during pregnancy (in utero) and is confirmed by a study using WHO child malnutrition and growth database as the average $z$ score of infants drop rapidly until the age of 2 years [43, 44]. Moreover, as the child grow, their needs for food and diverse diet increases as well as their requirements for calorie intake. Given the limited household resources, it is inevitable that with the growing body needs unfulfilled, stunting will result.

Child birth order is a significant risk factor for child health outcomes. The higher the birth order it is highly likely that the odds of a child being stunted increase 3 
folds, which is in line with the studies from India and Africa [45-47]. For India, the study explains that it is due to son preference and other reasons that mother's time allocation for child care is distributed among children in the household and competition for household food and non-food resources [47, 48]. Gender of a child is an important factor in stunting outcome; for example; Girls are facing a relatively higher burden of stunting. Girls have $27 \%$ higher odds of being severely stunted and $14 \%$ moderately stunted relative to boys. This finding is in line with the study from Nepal and Pakistan $[49,50]$ and from rural Thatta, Pakistan [31]' but in contrast with findings from Bhutan and Bangladesh $[5,51]$. This gender bias depicts the patriarchal nature of social setup, especially in Punjab, Pakistan. This is confirmed by a study of 61 countries ranking Pakistan secondhighest desired sex ratio for boys [52]. Furthermore gender discrimination and patriarchy leave female sex behind and neglect their fundamental rights like food. The findings of our study showed that children with diarrhea and less breast feeding at the earlier stages are more likely to be stunted. Similarly, rural residence (only in model 2 for moderate stunting) and smaller than the average size at birth are more likely to be stunted. These results are also reported in other studies [48, 53-55]. Mother and Fathers' higher education status is associated significantly with the less likelihood of a child of less than 5 years being stunted (moderate and severe) in Punjab, Pakistan. This result is in line with other studies from Asia and Africa $[28,34,56-58]$. Although the channels through which education of parents can affect child health outcomes are debated as it hypothesized that higher educated parents are relatively better endowed with (health care) knowledge and resources, relatively rich and better able to provide childcare $[28,59,60]$.

There are significant differences among the administrative divisions of the Punjab province. For example, Rawalpindi division has a significantly lower prevalence of moderate and severe stunting and compared to Rawalpindi, a child living in Lahore is twice likely to be stunted (moderate and severe) which is a surprising result given the relative socio-economic development of Lahore division compared to other administrative divisions of the province. One possible explanation is due to its metropolitan nature. Lahore division is relatively expensive to bear household food and non-food expenditure, especially for households with limited resources. Another possible reason can be that due to urbanization ( $82 \%$ population is urban in Lahore division) and at the same time it is highly congested (3500 person per square in Lahore district one of the highest in Punjab province and average household size 7.2 persons) and polluted city (poor living and unhygienic conditions) of the province which is detrimental for food absorption and health especially of children of younger ages [34, 39]. This result also points towards nutritional inequalities that exist among the administrative divisions of the Punjab province. This area of research needs further in depth empirical investigation. Mother's age at marriage, household size, pre and postnatal care, drinking water facility and region of residence are not significant predictors of moderate and severe stunting in any of the models estimated. However, communities with improved sanitation facilities have less likelihood of a child being moderately or severely stunted. This finding is in line with the previous results reported by other studies $[10,61,62]$. While analyzing the data from 172 Demographic and Health Surveys, the results showed that the likelihood of stunting in the household with sanitation facilities was relatively lower [63].

The difficulty of interpreting the OR has troubled many clinical researchers and epidemiologists for a long time (Chen et al. 2010). Hence, the transformation has been done based on Cohen (1988). The small effect indicates a weak association; the medium indicates a moderate association, and the large indicates the strong association between the two groups. The Cohen's $d$ statistic has shown clearly an improvement as some of the variables compared with the reference point has significant OR ratio indicated by less than $0.001 p$ values, however, they fall in the small (weak association) category indicated by Cohen's $d$ values less than 0.20 (Tables 2 and 3).

Our study has following limitation; as MICS data is cross sectional in nature hence we cannot interpret our results as causality. Second, we do not have data available for mother's diet and nutrition status which is an important variable of child health status. Finally, the MICS data is provincially representative but results cannot be generalizable at national level. Nevertheless, our findings are robust and setting stage for debate and policy discussions in one of the largest province of Pakistan. Hence, this study is an important step in formulating informed public policy decisions. Furthermore, there is no such analyses exist at sub national level in Pakistan that uses provincially representative large data set of children less than 5 years to analyze predictors of stunting.

\section{Conclusion}

The findings of this study confirm that the child specific, household, and district characteristics have reliable predicting power in the explanation of stunting (moderate and severe). Furthermore, the results also reveal that stark differences exist among household level, while at the community and district level, the variances decrease as we incorporate the explanatory variables. The findings of this study have strong policy implication, indicating 
focus should be on the household while making public policy. Nevertheless, policy makers should not forget the role of community (village) and district level factors in policy priorities.

\section{Abbreviations}

MICS: Multiple Indicator Cluster Survey; OLS: ordinary least square; PCA: Principal Component Analysis; SSUs: Secondary Sampling Units; UNICEF: United Nations Children's Fund; WASH: water, sanitation and hygiene; WHO: World Health Organization

\section{Acknowledgments}

We acknowledge Rachadapisek Sompote support for Postdoctoral Fellowship, Chulalongkorn University Thailand. Dr. Faisal Abbas acknowledge the receipt of the fellowship provided by Alexander Von Humboldt Foundation, Germany. Dr. Tahir acknowledges the receipt of faculty development fund (FDF) provided by higher Education Commission (HEC) Islamabad, Pakistan.

\section{Authors' contributions}

F.A and R. K performed the literature search and conceptualize this study. Data analysis was done by T. M with input from F.A. as well as interpretation of multilevel results. The manuscript was drafted by R. K and F. A. and reviewed by F. A, R. S and T.M. All the authors were involved in revision and editing of the draft. All authors critically reviewed the final manuscript and approved the draft for final submission.

\section{Funding}

This research paper receives no financial support.

\section{Availability of data and materials}

MICS data set is publically available online on the following link: http://mics. unicef.org/surveys

\section{Ethics approval and consent to participate}

This study is based on an analysis of cross sectional data available freely and publicly with all identifier information removed, no ethics approvals were required.

\section{Consent for publication}

Not Applicable.

\section{Competing interests}

The authors declare no conflict of interest.

\section{Author details}

${ }^{1}$ Department of Economics, University of Chitral, Chitral, Pakistan. ${ }^{2}$ American University of Beirut, Beirut, Lebanon. ${ }^{3}$ Department of Economics, School of Social Sciences and Humanities (S3H), National University of Sciences and Technology (NUST), Islamabad, Pakistan. ${ }^{4}$ Health Systems \& Policy Department, Health Services Academy, Islamabad, Pakistan. ${ }^{5}$ College of Public Health Sciences, Chulalongkorn University, Bangkok, Thailand.

Received: 30 September 2019 Accepted: 12 June 2020 Published online: 17 June 2020

\section{References}

1. Smith LC, Haddad L. Reducing child undernutrition: past drivers and priorities for the post-MDG era. World Dev. 2015;68:180-204.

2. United Nations Children's Fund (UNICEF). The state of the world's Children 2014.

3. Hoddinott J, Maluccio JA, Behrman JR, Flores R, Martorell R. Effect of a nutrition intervention during early childhood on economic productivity in Guatemalan adults. Lancet. 2008;371(9610):411-6.

4. Black RE, Victora CG, Walker SP, Bhutta ZA, Christian P, De Onis M, Uauy R. Maternal and child undernutrition and overweight in low-income and middle-income countries. Lancet. 2013;382(9890):427-51.

5. Aguayo VM, Badgaiyan N, Paintal K. Determinants of child stunting in the Royal Kingdom of Bhutan: an in-depth analysis of nationally representative data. Mat Child Nutr. 2015;11(3):333-45.
6. Deaton A, Drèze J. Food and nutrition in India: facts and interpretations. Econ and political weekly; 2009. p. 42-65.

7. World Health Organization. Physical status: The use and interpretation of Anthropometry. WHO technical report series 854. Geneva: World Health Organization; 1995. p. 224-228.

8. WHO. Physical Status: The use and interpretation of Anthropometry. WHO Technical Report Series, 854. Geneva: WHO; 1995.

9. Grantham-McGregor S, Cheung YB, Cueto S, Glewwe P, Richter L, Strupp B, International Child Development Steering Group, 2007. Developmental potential in the first 5 years for children in developing countries. Lancet. 2007;369(9555):60-70.

10. Cumming O, Cairncross S. Can water, sanitation and hygiene help eliminate stunting? Current evidence and policy implications. Mat Child Nutr. 2016;12: 91-105.

11. Schwarz NG, Grobusch MP, Decker ML, Goesch J, Poetschke M, Oyakhirome S, Kremsner PG. WHO 2006 child growth standards: implications for the prevalence of stunting and underweight-for-age in a birth cohort of Gabonese children in comparison to the Centers for Disease Control and Prevention 2000 growth charts and the National Center for Health Statistics 1978 growth references. Public Health Nutr. 2008;11(7):714-9.

12. World Health Organization (WHO), et al. Pub Health Nutr. 2006;11:714-9.

13. Bureau of Statistics Punjab, Planning \& Development Department, Government of the Punjab and UNICEF Punjab. Multiple Indicator Cluster Survey, Punjab 2014, Final Report. Lahore, Pakistan. Bureau of Statistics Punjab, Planning \& Development Department, Government of the Punjab and UNICEF Punjab. 2016.

14. Pakistan Demographic \& Health Survey (PDHS), 2012-13 ICF International USA: 2013.

15. Bhutta ZA, Gazdar H, Haddad L. Seeing the unseen: breaking the logjam of undernutrition in Pakistan. IDS Bulletin. 2013;44(3):1-9.

16. Cumming O, Cairncross S. Can water, sanitation and hygiene help eliminate stunting? Current evidence and policy implications. Matern Child Nutr. 2016; 12(Suppl 1):91-105.

17. Das JK, Achakzai ABK, Bhutta ZA. Stop stunting: Pakistan perspective on how this could be realized. Mat Child Nutr. 2016;12:253-6.

18. Kumar R, Abbas F, Tahir M, Somrongthong R. Prevalence and factors associated with underweight children: a populationbased subnational analysis from Pakistan. BMJ Open. 2019;9:e28972.

19. Adekanmbi VT, Kayode GA, Uthman OA. Individual and contextual factors associated with childhood stunting in Nigeria: a multilevel analysis. Mat Child Nutr. 2013;9:244-59.

20. Dewey KG, Begum K. Long-term consequences of stunting in early life. Mat Child Nutr. 2011;7:5-18.

21. Haile D, Azage M, Mola T, Rainey R. Exploring spatial variations and factors associated with childhood stunting in Ethiopia: spatial and multilevel analysis. BMC Pediatr. 2016;16:49.

22. Aguayo VM, Menon P. Stop stunting: improving child feeding, women's nutrition and household sanitation in South Asia. Mat Child Nutr. 2016;12:3-11.

23. Joe W, Rajaram R, Subramanian SV. Understanding the null-to-small association between increased macroeconomic growth and reducing child undernutrition in India: role of development expenditures and poverty alleviation. Mat Child nutr. 2016;12(S1):196-209.

24. Sharaf MF, Mansour El, Rashad AS. Child nutritional status in Egypt: A comprehensive analysis of socioeconomic determinants using a quantileregression approach. J Biosoc Sci. 2019;51(1):1-17.

25. Menon P. Childhood Undernutrition in South Asia: Perspectives from the Field of Nutrition. CESifo Econ Stud. 2012;58:274-95.

26. Kavosi E, Hassanzadeh Rostami Z, Kavosi Z, Nasihatkon A, Moghadami M, Heidari M. Prevalence and determinants of under-nutrition among children under six: a cross-sectional survey in Fars province, Iran. Int J Health Policy Manag. 2014;3:71-6.

27. Ahmed T, Hossain M, Mahfuz M, Choudhury N, Ahmed S. Imperatives for reducing child stunting in Bangladesh. Mat Child Nutr. 2016;12:242-5.

28. Vollmer S, Bommer C, Krishna A, Harttgen K, Subramanian SV. The association of parental education with childhood undernutrition in low-and middle-income countries: comparing the role of paternal and maternal education. Int J Epid. 2016:46(1):312-23.

29. Khan JR, Awan N, Misu F. Determinants of anemia among 6-59 months aged children in Bangladesh: evidence from nationally representative data. BMC Pediatr. 2016;16(1):3. 
30. Sand O, Kumar R, Shaikh TS, Somrogthong R, Hafeez A. Determinants of severe acute malnutrition among children under 5 years in a rural remote setting: A hospital based study from district Tharparkar-Sindh, Pakistan. Pak J Med Sci. 2018:34(2):260-5.

31. Nuruddin R, Hadden WC. Are pre-school girls more likely to be undernourished in rural Thatta, Pakistan?-a cross-sectional study. Int J Equity Health. 2015;14:151.

32. Shah SM, Selwyn BJ, Luby S, Merchant A, Bano R. Prevalence and correlates of stunting among children in rural Pakistan. Pediatr Int. 2003;45:49-53.

33. Mushtaq MU, Gull S, Khurshid U, Shahid U, Shad MA, Siddiqui AM. Prevalence and socio-demographic correlates of stunting and thinness among Pakistani primary school children. BMC Public Health. 2003;11:790.

34. Khalid H, Martin EG. Female-headed households associated with lower childhood stunting across culturally diverse regions of Pakistan: Results from a cross-sectional household survey. Mat Child Health J. 2017; 21(10):1967-84.

35. World Health Organization (WHO). Multi Center Growth Reference Study Group. WHO child growth standards based on length/height, weight and age. Acta Paediatrica. 2006;95(450):76-85.

36. World Food Programme.(WFP). Food Insecurity in Pakistan, Islamabad, Pakistan. Sustainable Development Policy Institute \& Swiss Agency for Development \& Cooperation 2009.

37. Snijders T, Bosker R. Multilevel modeling: An introduction to basic and advanced multilevel modeling; 1999.

38. Chen H, Cohen P, Chen S. How Big is a Big Odds Ratio? Interpreting the Magnitudes of Odds Ratios in Epidemiological Studies. J Comm Stat Simulation Computation. 2010;39(4):860-4.

39. Cohen J. Statistical Power Analysis for the Behavioral Sciences. Hillsdale: Erlbaum; 1998

40. Chirande L, Charwe D, Mbwana H, Victor R, Kimboka S, Issaka Al, Baines SK Dibley MJ, Agho KE. Determinants of stunting and severe stunting among under-fives in Tanzania: evidence from the 2010 cross-sectional household survey. BMC Pediatr. 2015;15(1):165.

41. Ahsan KZ, El Arifeen S, Al-Mamun MA, Khan SH, Chakraborty N. Effects of individual, household and community characteristics on child nutritional status in the slums of urban Bangladesh. Archives Public Health. 2017;75(1):9.

42. Gewa CA, Yandell N. Undernutrition among Kenyan children: contribution of child, maternal and household factors. Public Health Nutr. 2012;15(6): 1029-38.

43. De Onis M, Branca F. Childhood stunting: a global perspective. Mat Child Nutr. 2016;12(S1):12-26.

44. Victora CG, de Onis M, Hallal PC, Blössner M, Shrimpton R. Worldwide timing of growth faltering: revisiting implications for interventions. Pediatrics. 2010;125(3):e473-80.

45. Howell EM, Holla N, Waidmann T. Being the younger child in a large African Family: a study of birth order as a risk factor for poor health using the demographic and health surveys for 18 countries. BMC Nutr. 2016;2(1):61.

46. Horton S. Birth order and child nutritional status: evidence from the Philippines. Econ Dev and Cultural Change. 1998;36(2):341-54.

47. Jayachandran S, Pande R. Why are Indian children so short? The role of birth order and son preference. Am Econ Rev. 2017;107(9):2600-29.

48. Rahman MS, Howlader T, Masud MS, Rahman ML. Association of low-birth weight with malnutrition in children under 5 years in Bangladesh: Do mother's education, socio-economic status, and birth interval matter? Plos One. 2016;11(6):e0157814.

49. Tiwari R, Ausman LM, Agho KE. Determinants of stunting and severe stunting among under-fives: evidence from the 2011 Nepal Demographic and Health Survey. BMC Pediatr. 2014;14:239.

50. Hazarika G. Gender differences in children's nutrition and access to health care in Pakistan. J Dev Stud. 2000;37:73-92.

51. Chowdhury MRK, Rahman MS, Khan MMH, Mondal MNI, Rahman MM, Billah B. Risk factors for child malnutrition in Bangladesh: A multilevel analysis of a nationwide population-based survey. J Pediatr. 2016;172:194.

52. Bongaarts J. The implementation of preferences for male offspring. Pop Dev Rev. 2013;39(2):185-208.

53. Rakotomanana H, Gates GE, Hildebrand D, Stoecker BJ. The Determinants of Stunting in Children under 5 years in Madagascar. Mat Child Nutr. 2017;13: e12409.

54. Nkurunziza S, Meessen B, Korachais C. Determinants of stunting and severe stunting among Burundian children aged 6-23 months: evidence from a national cross-sectional household survey, 2014. BMC Pediatr. 2017;17(1):176.
55. Shinsugi C, Matsumura M, Karama M, Tanaka J, Changoma M, Kaneko S. Factors associated with stunting among children according to the level of food insecurity in the household: a cross-sectional study in a rural community of Southeastern Kenya. BMC Public Health. 2015;15(1):441.

56. Abuya BA, Ciera J, Kimani-Murage E. Effect of mother's education on child's nutritional status in the slums of Nairobi. BMC Pediatr. 2012;12:80.

57. Som S, Pal M, Bharati DP. Role of individual and household level factors on stunting: A comparative study in three Indian states. Ann Hum Bio. 2007;34: 632-46.

58. Dorsey JL, Manohar S, Neupane S, Shrestha B, Klemm RDW, West KP Jr. Individual, household, and community level risk factors of stunting in children younger than 5 years: Findings from a national surveillance system in Nepal. Matern Child Nutr. 2018;14(1):e12434.

59. Glewwe P. Why does mother's schooling raise child health in developing countries? Evidence from Morocco. J Hum Resour. 1999:34(1):124-59.

60. Gautam KP, Adhikari M, Khatri RB, Devkota MD. Determinants of infant and young child feeding practices in Rupandehi, Nepal. BMC Res Notes. 2016; 9(1):135.

61. Torlesse H, Cronin AA, Sebayang SK, Nandy R. Determinants of stunting in Indonesian children: evidence from a cross-sectional survey indicate a prominent role for the water, sanitation and hygiene sector in stunting reduction. BMC Public Health. 2016;16(1):669.

62. Spears D. How much international variation in child height can sanitation explain?. The World Bank; 2013.

63. Fink G, Günther I, Hill K. The effect of water and sanitation on child health: evidence from the demographic and health surveys 1986-2007. Int J Epidemiol. 2011;40(5):1196-204

\section{Publisher's Note}

Springer Nature remains neutral with regard to jurisdictional claims in published maps and institutional affiliations.
Ready to submit your research? Choose BMC and benefit from:

- fast, convenient online submission

- thorough peer review by experienced researchers in your field

- rapid publication on acceptance

- support for research data, including large and complex data types

- gold Open Access which fosters wider collaboration and increased citations

- maximum visibility for your research: over $100 \mathrm{M}$ website views per year

At BMC, research is always in progress.

Learn more biomedcentral.com/submissions 